\title{
Zur Philosophie des Kosmopolitismus
}

\section{Die Ursprünge des Kosmopolitismus}

Die Rede von dem Kosmopolitismus und - als eine Gegenposition - dem Kommunitarismus ist irreführend. Unter beiden Labels verbirgt sich jeweils ein ganzes Spektrum von philosophischen, politikwissenschaftlichen, politischen und weltanschaulichen Positionen. Eine umfassende kosmopolitische Konzeption liegt - soweit wir wissen - zum ersten Mal mit der griechischen Stoa vor. Es gab kynische Vorläufer (Diogenes von Sinope), die aber offenbar eher gegen einen engstirnigen Polis-Patriotismus Stellung nahmen, um die Einheit des Hellenentums zu bekräftigen. In der Stoa jedoch verbindet sich der politische Kosmopolitismus mit Ethik und Metaphysik auf beeindruckende Weise. Der Weltbürger der griechischen Stoa, ich denke dabei besonders an Chrysipp (von Arnim 1978, 1979), ist der in der vernünftigen, universellen Ordnung Beheimatete, der sich dieser vernünftigen Ordnung bewusst ist und der sich selbst in diese einfügt. Damit relativiert er seine eigene Bedeutung und findet als Weiser aus dieser Einsicht zu unerschütterlicher Seelenruhe und übernimmt doch Verantwortung, nicht nur für das eigene Leben, sondern für jeden Menschen unabhängig von seiner Herkunft, seiner Nation oder seinem Wohnort.

Die stoizistische Denkbewegung hat ihre Ursprünge noch in der Poliskultur der griechischen Klassik. Die politische Realität des Nationen und Kulturen übergreifenden Hellenismus ist nicht Ausgangspunkt, wohl auch nicht Folge, aber Begleitphänomen des stoizistischen Kosmopolitismus, so wie auch später das Imperium Romanum Begleitphänomen desselben ist. Die Einräumung des allgemeinen Bürgerrechts im Imperium Romanum im dritten Jahrhundert n. Chr. kann ebenso wie die frühchristliche Konzeption der civitas dei als politische Konsequenz des stoizistischen Denkens gelten. Für die christliche Kultur des Mittelalters aber ist die civitas dei ein fernes Ideal. Die diesseitige politische Ordnung ist kleinteilig, auch wenn sich über ihr in weiten Teilen Europas der imaginierte Nachfolger des Imperium Romanum in Gestalt des Heiligen Römischen Reiches Deutscher Nation etabliert hat. Es erscheint mir sinnvoll, auf diese Ursprünge des Kosmopolitismus in der Stoa $\mathrm{zu}$ verweisen, weil der politische Kosmopolitismus ohne die metaphysische Idee eines vernünftig geordneten Weltganzen eigene und neuartige Begründungslasten tragen muss. Die alte Opposition des griechischen Denkens zwischen den Universalien von Natur und den menschlichen Setzungen der jeweiligen politischen Gemeinschaft, der Gegensatz von physei und nomo, wird im stoizistischen Kosmopolitismus aufgehoben: Das von Natur aus Vernünftige wird zum Ordnungsprinzip der politisch-ethischen Sphäre. Der neuzeitliche und erst recht der zeitgenössische Kosmopolitismus muss ohne diese Einheit von Metaphysik, Ethik und Politik auskommen. 


\section{Varianten des Kosmopolitismus}

Ohne stoizistische Metaphysik und Ethik zerfällt der Kosmopolitismus in eine Vielfalt von Varianten. Diese reichen von der utopistischen, frühneuzeitlichen Idee des Weltstaates bis zur Vision der Auflösung aller Politik im globalen Markt, wie sie von Adam Smith bis zu den zeitgenössischen Marktradikalen propagiert wird. Der politische Kosmopolitismus strebt nach einer globalen Staatlichkeit und der mit dieser Staatlichkeit verbundenen Weltbürgerschaft. Der ursprünglich merkantilistische, kommerzielle oder ökonomische Kosmopolitismus versteht die Weltbürgerschaft als Teilhabe am globalen Markt (vgl. Hayek 1944, 1960; Friedman 1962). Er zielt auf die Auflösung nationaler Staatlichkeit, ohne diese durch globale Staatlichkeit ersetzen zu wollen. Der kulturelle und soziologische Kosmopolitismus (vgl. Beck 2004; Beck/Grande 2004) vertraut auf die Zunahme sozialer Interdependenzen und die Kreolisierung oder Hybridisierung kultureller Identitäten, um von einer Weltgesellschaft, die nationale Grenzen überwindet, sprechen zu können. Bisweilen verbindet sich der soziologische mit dem ökonomischen Kosmopolitismus gegen den politischen. Die Proponenten des modernen Nationalstaates betonen die Überwindung lokaler Identitäten, kultureller Herkünfte und weltanschaulicher Bindungen durch die Institutionen nationalstaatlicher Politik, die ein demos konstituiert, das im Rahmen demokratischer Institutionen legitimierte kollektive (politische) Entscheidungen trifft und damit das Repräsentationsprinzip realisiert.

Der politische Kosmopolitismus kann im Rahmen eines Mehrebenenmodells globaler Staatlichkeit die nationalstaatliche Identität besser integrieren als kulturelle und lokale Identitäten. Die Verbindung von republikanischer Selbstbestimmung und liberal-demokratischer bürgerlicher Freiheit im Nationalstaat soll im kosmopolitischen Föderalismus aufgehoben bleiben, während der ökonomische ebenso wie der kulturell-soziologische Kosmopolitismus das nationalstaatliche Politikmodell grundsätzlich überwinden will und seine Einbettung in eine global-föderalistische Ordnung ablehnt.

\section{Kosmopolitismus und Universalismus}

Der Kosmopolitismus steht gegen den Kommunitarismus im weitesten Sinne. Hier wird unter »Kommunitarismus« ebenfalls ein ganzes Spektrum von Positionen verstanden, das von unitaristischen und nationalistischen - etwa Alasdair MacIntyre (1981, 1988) - bis zu liberalen und sozialdemokratischen - etwa Michael Walzer (1983, 1987, 2005) - Varianten reicht. Der kommunitaristischen Gegenposition gemeinsam ist die Skepsis gegenüber anthropologischen Gleichheitsannahmen. Der Mensch ist, wie er ist, Teil einer kulturellen, lokalen oder politischen Gemeinschaft. Radikale Vertreter des Kommunitarismus wie etwa Mary Douglas (1986) behaupten sogar, dass die kollektiven Identitäten die eigentlichen Akteure seien, während das jeweilige Individuum nur scheinbar handle. Die Idee eines autonomen Subjektes, die Vorstellung einer kulturunabhängigen Rationalität ist allen Varianten des Kommuni- 
tarismus fremd. Sie einigt andererseits alle Varianten des Kosmopolitismus. Mit anderen Worten: der Kosmopolitismus setzt auf das Individuum, das sich aus seiner kulturellen, sozialen und politischen Situierung lösen kann und sich in seinen ethischen wie politischen Überzeugungen an universellen Prinzipien orientiert. Dieser Gegensatz scheint unüberwindlich zu sein.

Entgegen der üblichen, schroffen Gegenübersetzung von kosmopolitischem Universalismus und kommunitaristischem Partikularismus sind beide unverzichtbaren Elemente jeder Normativität - Universalität und Partikularität - miteinander vereinbar. Das Argument kann hier nicht im Detail entwickelt werden (siehe dazu NidaRümelin 1999: Kap. 13), aber die zentrale Unterscheidung zwischen der Universalität der normativen Überzeugungen einerseits und der Partikularität ihrer Begründung andererseits soll hier wenigstens kurz erläutert werden. Normative (politische oder moralische) Überzeugungen ähneln insoweit empirischen oder deskriptiven Überzeugungen, als sie einen universellen Geltungsanspruch haben - sonst wären es keine Überzeugungen. Ein Relativismus, der ernsthaft die Auffassung vertritt, dass Überzeugungen jeweils versteckt einen Index trügen, also nur für diejenigen Personen gelten, die sie haben, bzw. für Personen einer bestimmten Kultur, einer bestimmten Herkunft etc., steht mit der Grammatik und der Interaktionspraxis unserer Lebenswelt im Konflikt. Natürlich kann man eine Irrtumstheorie der normativen Sprache unterstellen (vgl. Mackie 1977), aber damit eskamotiert sich die Theorie aus den kulturellen, sozialen und politischen Begründungszusammenhängen. Sie wird extern, verliert ihre kritische Kraft und wird a limine unverständlich (vgl. Walzer 1987). Normative Überzeugungen haben, wie deskriptive, einen universellen Geltungsanspruch. Der Universalismus ist in die Logik der normativen Sprache eingelassen.

Dies schließt aber keineswegs die Berücksichtigung von Partikularitäten aus. Eltern haben gegenüber ihren Kindern andere Pflichten als gegenüber den Kindern anderer Menschen. Diese normative Überzeugung ist den meisten Menschen gemeinsam und sie wird mit einem universellen Geltungsanspruch verbunden. Grundsätzlich sind besondere Pflichten gegenüber der eigenen kulturellen Gemeinschaft, den Mitgliedern derselben Nation oder Kultur mit einem normativen Universalismus vereinbar. Diese besonderen Pflichten gelten dann eben universell. Die Begründung solcher partikularen Bindungen muss aber wieder im universalistischen Modus erfolgen. Sie darf nicht willkürliche Eigenschaften benennen, sondern moralisch relevante. Wenn etwa angenommen werden kann, dass es dem Gedeihen der Menschheit gut tut, wenn es besondere Verantwortlichkeiten im sozialen Nahbereich gibt, dann erscheinen diese universell begründbar.

Der anthropologische Kosmopolitismus geht von der grundsätzlichen Gleichheit aller Menschen, ihrem gleichen moralischen Status und dem sich daraus ergebenden normativen Gebot gleichen Respekts aus (vgl. Habermas 1999). Daraus ergibt sich mitnichten logisch zwingend die Ablehnung besonderer Bindungen lokaler, kultureller, religiöser oder nationaler Zugehörigkeiten. Diese Zugehörigkeiten können als Kooperationsgemeinschaften verstanden werden, deren Existenz auch aus einer kosmopolitischen oder humanistischen Perspektive sinnvoll erscheint. In der 
Geschichte des kosmopolitischen Denkens haben seine gemäßigteren Vertreter sich nicht grundsätzlich gegen partikulare Bindungen der Familien, der lokalen Gemeinschaft, der Kultur oder der Nation gestellt.

Auch wenn normative Überzeugungen einen zwingend universellen Geltungsanspruch haben, so sind ihre Begründungen doch jeweils partikular, sie enden in den vorfindlichen epistemischen Bedingungen der Verständigungsgemeinschaft. Das Descartessche Programm einer wissenschaftlichen Rationalität, die rationale von irrationalen Überzeugungen scheidet und die ihr Begründungsfundament in nicht mehr bezweifelbaren und selbstevidenten Axiomen hat, lässt sich nicht durchführen. Die Wissenschaft selbst ist auf die unsere Lebenswelt bestimmenden Überzeugungssysteme angewiesen. Unser Zugang zu den Gegenständen der alltäglichen Erfahrung wird nicht erst durch die Wissenschaft gestiftet, die wechselseitige Zuschreibung von Intentionen, Einstellungen und Überzeugungen geht der Wissenschaft notwendig voraus und imprägniert diese. Eine in dem Sinne externe Kritik lebensweltlichen Orientierungswissens, sei sie normativer oder deskriptiver Natur, ist aus epistemologischen Gründen nicht möglich. Jede Kritik kommt von innen, d. h. sie knüpft an zentrale Elemente lebensweltlicher Wissensbestände an und weist auf Widersprüche, auf Inkohärenzen hin. Diese philosophische Perspektive ist nicht ohne Bedeutung für den politischen Kosmopolitismus.

\section{Kosmopolitische Zivilgesellschaft als Kooperation}

Es scheint mir sinnvoll zu sein, die Zivilgesellschaft über den Begriff der Kooperation zu fassen. Kooperatives Verhalten ist eine besondere Form des Verhaltens, für die zwei Elemente charakteristisch sind: Kooperation verlangt zum einen, dass die an der Kooperation Beteiligten im Großen und Ganzen korrekte Einschätzungen ihrer jeweiligen individuellen Interessenlage haben, d. h. sie setzt ein gewisses Maß an Empathie voraus. Was Kooperation jeweils ist, wird über die individuellen Interessen, die von der Interaktion berührt sind, bestimmt. Zum anderen aber kann eine kooperative Praxis nur Erfolg haben, wenn die einzeln an ihr Beteiligten darauf verzichten, ihre je individuellen Interessen zu optimieren. Sie müssen bereit sein im Sinne ihrer eigenen Interessen »suboptimal« zu agieren, indem sie jeweils ihren Beitrag zu einer gemeinsamen kooperativen Praxis leisten. Diesen Beitrag leisten sie in der Erwartung, dass andere ebenfalls ihren Beitrag leisten, wohl wissend, dass es jeweils eine andere individuelle Strategie für jede Person, die an der Kooperation beteiligt ist, gäbe, die für diese individuell günstiger wäre. Die Interaktionsform der Kooperation kann man daher als eine Vermittlung zwischen zwei Standpunkten dem partikularen Interessen-Standpunkt und dem universellen moralischen Standpunkt - verstehen.

Mit diesem Begriff der Kooperation lassen sich drei große Bereiche menschlicher Gesellschaften gegeneinander abgrenzen: Der Markt, der Staat und die Zivilgesellschaft. Während der Markt lediglich eigeninteressierte Optimierer als Konsumenten und Produzenten, als Anbieter und Nachfrager postuliert, beruhen staatliche Institu- 
tionen auf dem Prinzip der hierarchischen Verantwortung, der Weisungsunterstellung und des weisungsgebundenen Handelns. Idealtypisch hat Kooperation auf dem Markt keinen Platz; z. B. unterbinden Kartellgesetze Kooperationen sofern sie die Konkurrenzsituation verzerren. Idealtypisch kommen auch staatliche Institutionen ohne Kooperation aus, da Kooperationen, welche die administrativen HierarchieLinien konterkarieren, traditionell als ein bedrohliches Phänomen wahrgenommen werden. Kooperation ist dagegen diejenige Interaktionsform, die die Zivilgesellschaft trägt. Als Bürger handeln die Mitglieder einer Zivilgesellschaft nicht im privaten Interesse, sondern für gemeinsame bürgerschaftliche Zielsetzungen und Programme (vgl. Kaldor 2003). Die individuellen Interessenlagen bleiben jedoch Referenzpunkte für die bürgerschaftliche Begründung dieser Zielsetzungen und Programme.

Zwischen Zivilgesellschaft und Demokratie besteht ein wechselseitiges Stützungsverhältnis. Demokratische Institutionen stabilisieren die Kooperationsformen der Zivilgesellschaft und umgekehrt. Der kollektive Akteur, der für die traditionelle Demokratietheorie eine so zentrale Rolle spielte, ist prinzipiell - möglicherweise aber nicht unter den gegebenen pragmatischen Bedingungen - verzichtbar. Von daher ist z. B. die Staatlichkeit der Europäischen Union oder die einer Weltrepublik mit dem Konzept der Demokratie als Kooperation vereinbar. Nur um Missverständnisse auszuschließen, sei angemerkt, dass Staatlichkeit ganz ohne Markt und ganz ohne Zivilgesellschaft ebenso wenig vorstellbar ist, wie ein ökonomischer Markt ganz ohne staatliche Garantien, etwa des Vertragsrechtes, und zivilgesellschaftliche Kooperationen. Und auch eine Zivilgesellschaft pur ohne Markt und ohne Staat wird immer eine anarchistische Chimäre bleiben.

\section{Welcher Kosmopolitismus?}

Wenn nicht alle Zeichen trügen, wird es in Zukunft nicht um die Frage gehen »Kosmopolitismus - ja oder nein?«, sondern um die Frage »Welcher Kosmopolitismus?«. Dies gilt jedenfalls dann, wenn man »Kosmopolitismus « im weiteren Sinne fasst, d. h. als jede Form von Weltgesellschaft, die Weltbürgerschaft einschließt. In diesem weiteren Sinne verstanden, stehen sich drei kosmopolitische Konzeptionen gegenüber: Die erste hat ihren Ursprung in der schottischen Aufklärung und im Merkantilismus und zielt auf eine Weltgesellschaft der Marktbürger. Adam Smith (1976) hat an seiner Auffassung keinen Zweifel gelassen, dass diese Form der Weltbürgerschaft zu einer Auflösung der Nationalstaaten und generell von staatlichen Institutionen führen wird bzw. jedenfalls dazu führen sollte. Der zeitgenössische Neo-Liberalismus (vgl. Hayek 1944, 1960; Friedman 1962), den man besser als »Marktradikalismus « bezeichnen sollte, zielt ebenfalls auf einen Abbau von Staatlichkeit generell, nicht nur nationaler Staatlichkeit. Der soziologische (kulturelle) Kosmopolitismus diagnostiziert die Auflösung überkommener nationaler Identitäten und begrüßt das, was gelegentlich als Kreolisierung bezeichnet wurde, also die Überlappungen, Vermengungen und Multiplizierungen kultureller und sozialer Identitäten. Die Zugehörig- 
keit des Einzelnen zu mehreren Identitäten und die Präsenz dieser Identitäten in jedem Nationalstaat konstituiert eine Weltgesellschaft auch für diejenigen, deren Lebensform nach wie vor ortsfest geblieben ist. Der soziologische teilt mit dem ökonomischen Kosmopolitismus die Kritik nationaler Staatlichkeit speziell und tradierter Staatlichkeit generell. Die Karriere des Begriffs »Governance « in der politischen Soziologie ist dafür symptomatisch. An die Stelle politischer Entscheidungsverfahren und rechtlich verfasster Institutionen tritt das Paradigma ausgehandelter, möglichst konsensualer Steuerung. Je nach Akzentuierung kann dabei die globale Zivilgesellschaft etwa in Gestalt von NGOs zu einem zentralen Akteur werden. Dieser anti-etatistische Zug des ökonomischen und des soziologischen Kosmopolitismus mobilisiert Verteidiger des Nationalstaates ganz unterschiedlicher politischer Provenienz: Liberale wie Ralf Dahrendorf (2003), Konservative wie Alasdair MacIntyre (1981, 1988), Linke wie Chantal Mouffe (2005). Ihnen geht es in unterschiedlichen Formen um die Bewahrung des politisch verfassten demos und damit der Idee kollektiver politischer Selbstbestimmung. Insbesondere in der französischen Staatstradition wird dabei der nationalstaatlich verfasste demos als Ausdruck kosmopolitischer, die lokalen kulturellen und religiösen Grenzen überwindender Identität angesehen, die sich mit einem anthropologischen und ethischen Kosmopolitismus nahtlos verbinden lässt. Sowohl in den gegenwärtigen politischen Konfliktlinien, etwa hinsichtlich der europäischen Verfassung, als auch in der politischen Theorie zeichnet sich immer deutlicher eine Verbindung von nationaler Staatlichkeit und politischem Kosmopolitismus ab, die sich gegen die Auflösung des Primats der Politik und die Aufgabe der Idee kollektiver politischer Selbstbestimmung richtet. Die am weitesten entwickelten Konzeptionen des politischen Kosmopolitismus sind - insofern wenig überraschend - so verfasst, dass sie eingebetteten Nationalstaaten eine zentrale Rolle zuerkennen (vgl. Archibugi 2003; Archibugi/Held 1995; Höffe 2000).

Wenn man Demokratie als eine durch politische Institutionen gestützte Form der Zivilgesellschaft versteht, dann stünde der politische Kosmopolitismus nicht mehr vor der Alternative Nationalstaat oder Weltstaat, sondern könnte sich einer Mehrebenenstaatlichkeit öffnen, die nationalstaatliche Souveränitäten in den Grenzen allgemein anerkannter Menschen- und Bürgerrechte bewahrt. Der Aufbau internationaler Gerichtsbarkeit und die Sanktionierung über nationalstaatliche und subnationalstaatliche Behörden (etwa Landesbehörden) ist keine exotische Idee, sondern schon aus dem europäischen Einigungsprozess vertraut.

Ein »weicher« Kosmopolitismus des beschriebenen Typs eignet sich nicht für die oft befürchtete Instrumentalisierung im Kampf um die Hegemonie des Westens. Auch wenn Normativität ohne universellen Geltungsanspruch nicht zu haben ist, so sind die normativen Begründungen in den Partikularitäten der Lebensform und der Kultur verankert. Es hat sich in den vergangenen Dekaden deutlich gezeigt, dass die Menschenrechte - und damit meine ich nicht nur die der ersten Generation, also die individuellen Freiheitsrechte westlicher Verfassungen, sondern auch soziale und kulturelle Rechte, wie sie in den Menschenrechtskonventionen der Vereinten Nationen enthalten sind - nicht lediglich ein Produkt westlicher Kultur sind, sondern auch in anderen kulturellen, religiösen und sozialen Kontexten begründet werden können. 
So ist der zentrale Wert der Menschenwürde und der Selbstachtung in allen großen Kulturregionen der Welt tief verankert, ebenso wie Hilfspflichten, Kooperationsgebote und Freiheitsansprüche. Entgegen mancher Erwartung hat sich die demokratische Staatsform, wenn auch in einem breiten Spektrum von Gestaltungsvarianten, als kulturell in hohem Maße anpassungsfähig erwiesen. Hier kämpfen nicht die Weberschen Götter ihren ewigen Kampf miteinander (Weber 1973), sondern die Übereinstimmung in normativen Grundfragen der Politik ist hinreichend groß, um einen globalen Verständigungsprozess auch über Institutionalisierungen, wie etwa ein Weltstrafgericht, zuzulassen. Der anthropologische und ethische Universalismus, der den politischen Kosmopolitismus erst hat entstehen lassen, legitimiert weder einen militärisch gestützten Demokratie-Export, noch humanitär motivierte Interventionen. Daher gilt auch umgekehrt: man kann Universalist und Kosmopolitin sein, ohne humanitäre Interventionen und die amerikanische Politik des Demokratie-Exports gutzuheißen (vgl. Nida-Rümelin 2006).

\section{Literatur}

Archibugi, Daniele/Held, David (Hrsg.) 1995: Cosmopolitan Democracy: An Agenda for a New World Order, Cambridge.

Archibugi, Daniele (Hrsg.) 2003: Debating Cosmopolitics, London.

Beck, Ulrich 2004: Der kosmopolitische Blick oder: Krieg ist Frieden, Frankfurt a. M.

Beck, Ulrich/Grande, Edgar 2004: Das kosmopolitische Europa. Gesellschaft und Politik in der Zweiten Moderne, Frankfurt a. M.

Dahrendorf, Ralf 2003: Auf der Suche nach einer neuen Ordnung. Eine Politik der Freiheit für das 21. Jahrhundert, München.

Douglas, Mary 1986: How Institutions Think: The Frank W. Abrams Lectures, Syracuse, NY. Friedman, Milton 1962: Capitalism and Freedom, Chicago, IL.

Habermas, Jürgen 1999: Die Einbeziehung des Anderen. Studien zur politischen Theorie, Frankfurt a. M.

Hayek, Friedrich August 1944: The Road to Selfdom, London.

Hayek, Friedrich August 1960: The Constitution of Liberty, London.

Höffe, Otfried 2000: Vision: föderale Weltrepublik (Akademiegespräche im Bayerischen Landtag), München.

Kaldor, Mary 2003: Global Civil Society: An Answer to War, Cambridge, MA.

MacIntyre, Alasdair 1981: After Virtue: A Study in Moral Theory, Notre Dame, IN.

MacIntyre, Alasdair 1988: Whose Justice, Which Rationality?, Notre Dame, IN.

Mackie, John 1977: Ethics: Inventing Right and Wrong, Harmonsworth.

Mouffe, Chantal 2005: Eine kosmopolitische oder eine multipolare Weltordnung?, in: Deutsche Zeitschrift für Philosophie 2005: 53, 69-81.

Nida-Rümelin, Julian 1999: Demokratie als Kooperation, Frankfurt a. M.

Nida-Rümelin, Julian 2006: Demokratie und Wahrheit, München.

Smith, Adam 1976: An Inquiry into the Nature and Causes of the Wealth of Nations, Nachdruck von 1776, Chicago, IL.

von Arnim, Hans (Hrsg.) 1979: Stoicorum veterum fragmenta. Band 2: Chrysippi fragmenta logica et physica, 3., unveränderter Nachdruck von 1903, München.

von Arnim, Hans (Hrsg.) 1978: Stoicorum veterum fragmenta. Band 3: Chrysippi fragmenta moralia. Fragmenta successorum Chrysippi, 3., unveränderter Nachdruck von 1903, München. 
Walzer, Michael 1983: Spheres of Justice: A Defense of Pluralism and Equality, New York, NY.

Walzer, Michael 1987: Interpretation and Social Criticism, Cambridge, MA.

Walzer, Michael 2005: Politics and Passion, Yale, MA.

Weber, Max 1973: Der Sinn der »Wertfreiheit« der soziologischen und ökonomischen Wissenschaften, in: Weber, Max: Gesammelte Aufsätze zur Wissenschaftslehre, 4., erneut durchgesehene Auflage, herausgegeben von Johannes Winckelmann, Tübingen, 489520 . 